\title{
Influence of Local Magnetic Fields on P-Doped Si Floating Zone Melting Crystal Growth in Microgravity
}

K. Li* and W. R. Hu

National Microgravity Laboratory/ CAS, Institute of Mechanics, CAS, 15 Zhong Guan Cun Road, Beijing 100080, China

ABSTRACT: A two-dimensional axisymmetric numerical model is presented to study the influence of local magnetic fields on P-doped Si floating zone melting crystal growth in microgravity. The model is developed based on the finite difference method in a boundary-fitted curvilinear coordinate system. Extensive numerical simulations are carried out, and parameters studied include the curved growth interface shape and the magnetic field configurations. Computed results show that the local magnetic field is more effective in reducing the impurity concentration nonuniformity at the growth interface in comparison with the longitudinal magnetic field. Moreover, the curved growth interface causes more serious impurity concentration nonuniformity at the growth interface than the case with a planar growth interface.

\section{Introduction}

The floating zone method is a crucible free process used for the melting growth of high quality single crystals. The microgravity environment is beneficial to the large size crystal growth by the floating zone method because the melt zone is confined by the melt surface tension. In microgravity, the thermocapillary convection induced by the melt surface tension gradient is dominant in the melt zone while the buoyancy driven convection is greatly reduced. The thermocapillary convection may be unsteady and thus induces the impurity striations in the grown crystal. Usually an externally applied Iongitudinal magnetic field is used to dampen the unsteady thermocapillary convection. ${ }^{1-4}$ With a strong magnetic field, the thermocapillary convection in the central region of the melt zone is suppressed and the impurity striations in the according part of the grown crystal are eliminated. ${ }^{4}$ H owever, the longitudinal magnetic field has no direct influence on the flow al ong the magnetic field lines such as the flow along the nondeformed free surface; thus, the relatively strong thermocapillary convection still occupies the region near the free surface. Such a flow field pattern induces large radial impurity macrosegregation in the grown crystal. ${ }^{4}$ So it is necessary to improve the flow field pattern in the melt zone to reduce the impurity concentration nonuniformity at the growth interface by an optimal configuration of the applied magnetic field. . $^{3,5}$

Recently, numerical studies ${ }^{6,7}$ were conducted to evaluate the influence of the local magnetic fields on the P-doped Si floating zone melting crystal growth in microgravity. Two-dimensional (2D) axisymmetric crystal growth models with the planar phase change interface simplification were used in the above studies. The local magnetic fields produced by the electrical coils located near the melt zone were specially designed to damp the thermocapillary convection in the regions near the free surface, and the melt flow in the central region

* To whom correspondence should be addressed. School of Mechanical and Materials Engineering, Washington State University, Pullman, WA 99164. E-Mail: kli@mail.wsu.edu. of the melt zone was reduced as a result. The computed results illustrate that with the more uniform damped flow field pattern, the approach of the local magnetic fiel d can reduce the impurity concentration nonuniformity at the growth interface significantly. However, the planar interface shape is the simplification of the real crystal growth process. To improve the numerical model to relate the practice more closely, the phase change interfaces coupled with heat and mass transfer in the melt zone are calculated in the present study. The numerical model was developed based on the finite difference method in a boundary-fitted curvilinear coordinate system. The problem statement is presented in the next section, the numerical method is given in Section 3, and the numerical results and discussion are given in Section 4. The last section is the conclusion.

\section{Problem Statement}

Figure 1 schematically illustrates the 2D axisymmetric model of the P-doped Si fl oating zone melting crystal growth in microgravity. The melt zone is floating between the melting interface $z_{1}(r)$ and the growth interface $z_{2}(r)$, respectively. The radius of the computation domain is $\mathrm{R}_{0}$ because the free surface deformation in microgravity is neglected here. ${ }^{6,7}$ The dependence of the melting temperature on the impurity concentration is not included because the considered impurity is dilute. The ambient temperature distribution is assumed to be a Gaussian distribution ${ }^{5}$ as follows

$$
\mathrm{T}_{\mathrm{a}}(\mathrm{z})=\mathrm{T}_{\infty}+\left(\mathrm{T}_{\mathrm{p}}-\mathrm{T}_{\infty}\right) \exp \left[-(\mathrm{z}-0.5 \mathrm{~L})^{2} / \mathrm{a}^{2}\right]
$$

The length of the computation domain is chosen to be long enough to ensure that the far end temperature $T_{\infty}$ can be assumed to be a constant.

In the present study, the pseudo-steady state model $(\text { PSSM })^{8}$ is adopted. The feed rod is fed into the melt zone, and the grown crystal is withdrawn from the above at the speed that conserves the mass of the system. Because the magnetic Reynolds number is very small for semiconductors, the induced magnetic field can be 


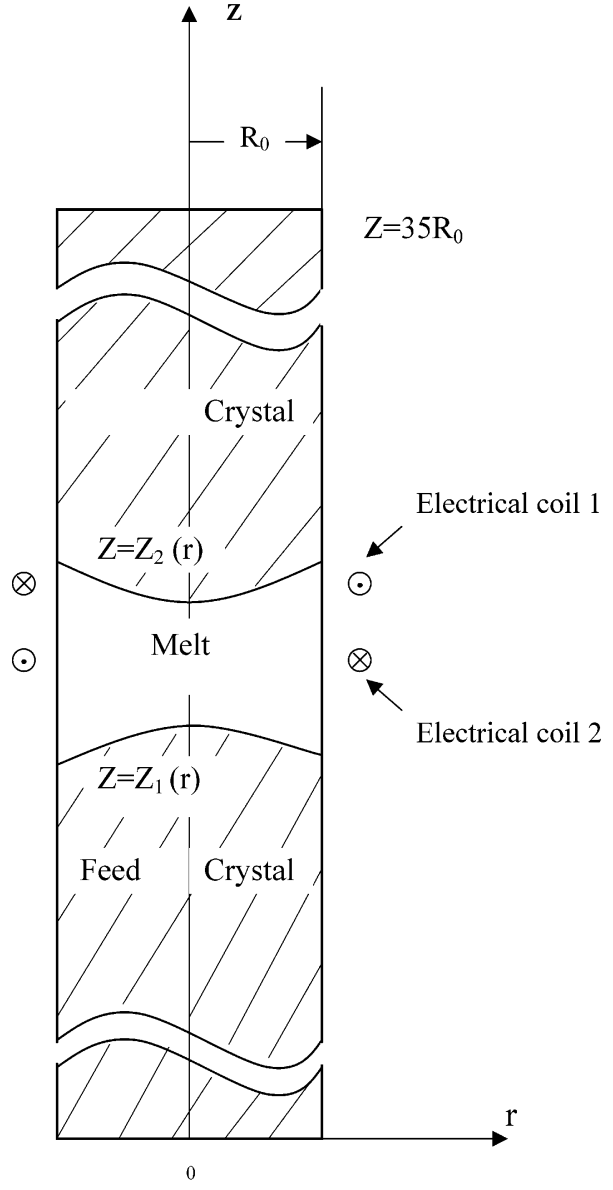

Figure 1. Schematic diagram of a floating zone melting crystal growth model in microgravity.

Table 1. Related Coefficients $a_{i}, b_{i}, c_{i}$, and $d_{i}$

\begin{tabular}{cllll}
\hline$\phi_{i}$ & \multicolumn{1}{c}{$a_{i}$} & $b_{i}$ & $c_{i}$ & \multicolumn{1}{c}{$d_{i}$} \\
\hline$\psi(i=1)$ & 0 & $1 / r$ & 1 & $\omega$ \\
$\omega(i=2)$ & $P e / r$ & $\operatorname{Pr} / r$ & $r$ & $\mathrm{Ha}^{2} \operatorname{Pr}\left[\frac{\partial}{\partial z}\left(j_{\theta} B_{z}\right)+\frac{\partial}{\partial r}\left(j_{\theta} B_{r}\right)\right]$ \\
$T(i=3)$ & $P e\left(C_{p s} P e\right)$ & $r\left(k_{s} r\right)$ & 1 & 0 \\
$C(i=4)$ & $\operatorname{Pes}_{s}$ & $r$ & 1 & 0
\end{tabular}

neglected and the induced electric current is adopted as zero in this case. The thermocapillary convection in the melt zone is assumed steady and axisymmetric, and only heat transfer is considered in solid phase. In the present study, the streamfunction $\psi$ and vorticity $\omega$ are defined as follows ${ }^{5,9}$

$$
\mathrm{u}=-\frac{1}{\rho \mathrm{r}} \frac{\partial \psi}{\partial \mathrm{z}}, \mathrm{v}=\frac{1}{\rho \mathrm{r}} \frac{\partial \psi}{\partial \mathrm{r}}, \omega=\frac{\partial \mathrm{u}}{\partial \mathrm{z}}-\frac{\partial \mathrm{v}}{\partial \mathrm{r}}
$$

Thus, the nondimensional governing equations are written in the general form:

$$
\begin{aligned}
& \frac{\partial}{\partial r}\left(a_{i} \phi_{i} \frac{\partial \psi}{\partial z}\right)-\frac{\partial}{\partial z}\left(a_{i} \phi_{i} \frac{\partial \psi}{\partial r}\right)+\frac{\partial}{\partial r}\left[b_{i} \frac{\partial c_{i} \phi_{i}}{\partial r}\right]+\frac{\partial}{\partial z}\left[b_{i} \frac{\partial c_{i} \phi_{i}}{\partial z}\right]+ \\
& \mathrm{d}_{\mathrm{i}}=0 \text { (2.3) }
\end{aligned}
$$

The related parameter definitions in eq 2.3 are listed in Table 1. In deriving the above equations, scales are used as follows: $R_{0}$ for length, $U_{0}=\left|\sigma_{T}\right| \Delta T / \rho v$ for velocity, $\omega_{0}=\left(\mathrm{U}_{0} / \mathrm{R}_{0}\right)$ for vorticity, $\psi_{0}=\left(\psi / \rho_{0} \mathrm{U}_{0} \mathrm{R}_{0}{ }^{2}\right)$ for streamfunction, $p_{0}=\rho_{0} U_{0}{ }^{2}$ for pressure, $C_{0}$ for concentration, $\mathrm{B}_{0}=\mu_{\mathrm{r}} \mu_{0} \mathrm{l}_{0} / \pi \mathrm{R}_{0}$ for magnetic field, and $\Delta \mathrm{T}=$
$\left(T_{p}-T_{0}\right)$ for temperature while the definition of the nondimensional temperature is $\left(T-T_{0}\right) / \Delta T$. The nondimensional quantities and parameters are defined as follows

$$
\begin{array}{r}
\operatorname{Pr}=\frac{v}{\kappa}, \mathrm{Pe}=\frac{\mathrm{U}_{0} \mathrm{R}_{0}}{\kappa}, \mathrm{Pe}_{\mathrm{s}}=\frac{\mathrm{U}_{0} \mathrm{R}_{0}}{\mathrm{D}}, \mathrm{Ma}=\frac{\sigma_{\mathrm{T}} \mathrm{R}_{0} \Delta \mathrm{T}}{\rho v^{2}}, \\
\mathrm{Ra}=\frac{\sigma \epsilon \mathrm{R}_{0}(\Delta \mathrm{T})^{3}}{\mathrm{k}}, \mathrm{Ra}_{\mathrm{s}}=\frac{\rho \epsilon_{\mathrm{s}} \mathrm{R}_{0}(\Delta \mathrm{T})^{3}}{\mathrm{k}}, \\
\mathrm{Ha}=\mathrm{B}_{0} \mathrm{R}_{0} \sqrt{\sigma_{\mathrm{e}} \rho v}, \mathrm{Ste}=\frac{\Delta \mathrm{H}}{\mathrm{C}_{\mathrm{p}} \Delta \mathrm{T}}
\end{array}
$$

The boundary conditions for the configuration considered are determined by the physical constraints and are detailed below.

(a) Melt Zone Region. (i) Along the growth axis, $r=0$ :

$$
\psi=0, \omega=0, \frac{\partial T}{\partial r}=0, \frac{\partial C}{\partial r}=0
$$

$\psi=0$ is adopted here as the reference.

(ii) Along the free surface, $r=1$ :

$$
\begin{aligned}
\psi= & 0.5 \mathrm{~V}_{\mathrm{p}}, \omega=-\frac{\operatorname{MaPr}}{\operatorname{Pe}} \frac{\partial \mathrm{T}}{\partial \mathrm{z}}, \frac{\partial \mathrm{C}}{\partial \mathrm{r}}=0, \\
& -(\mathbf{n} \cdot \nabla \mathrm{T})=\operatorname{Ra}\left\{\left[\mathrm{T}+\mathrm{T}_{0}^{*}\right]^{4}-\left[\mathrm{T}_{\mathrm{a}}(\mathrm{z})+\mathrm{T}_{0}^{*}\right]^{4}\right\}
\end{aligned}
$$

(iii) Along the melting interface, $z=z_{1}(r)$ :

$$
\begin{aligned}
\psi=0.5 \mathrm{~V}_{\mathrm{p}} \mathrm{r}^{2}, \omega= & \frac{\partial \mathrm{u}}{\partial \mathrm{z}}-\frac{\partial \mathrm{v}}{\partial \mathrm{r}}, \mathrm{T}=0, \\
& \mathbf{n} \cdot \nabla \mathrm{C}=\operatorname{Pe}_{\mathrm{S}} \mathrm{V}_{\mathrm{p}}(\mathrm{C}-1)\left(\mathbf{n} \cdot \mathbf{e}_{\mathbf{z}}\right)
\end{aligned}
$$

(iv) Along the growth interface, $z=z_{2}(r)$ :

$$
\begin{aligned}
\psi=0.5 \mathrm{~V}_{\mathrm{p}} \mathrm{r}^{2}, \omega=\frac{\partial \mathrm{u}}{\partial \mathrm{z}}-\frac{\partial \mathrm{v}}{\partial \mathrm{r}}, \mathrm{T}=0, \\
\mathbf{n} \cdot \nabla \mathrm{C}=\mathrm{Pe}_{\mathrm{s}} \mathrm{V}_{\mathrm{P}}\left(1-\mathrm{k}_{0}\right) \mathrm{C}\left(\mathbf{n} \cdot \mathbf{e}_{\mathbf{z}}\right)
\end{aligned}
$$

(b) Solid Regions. (i) Along the growth axis, $r=0$ :

$$
\frac{\partial T}{\partial r}=0
$$

(ii) Along the surface, $r=1$ :

$$
-(\mathbf{n} \cdot \nabla \mathrm{T})=\mathrm{Ra}_{\mathrm{s}}\left\{\left[\mathrm{T}+\mathrm{T}_{0}^{*}\right]^{4}-\left[\mathrm{T}_{\mathrm{a}}(\mathrm{z})+\mathrm{T}_{0}^{*}\right]^{4}\right\}
$$

(iii) Far ends

$$
\mathrm{z}=0 \text { and } \mathrm{z}=35: \mathrm{T}=\mathrm{T}_{\infty}
$$

In addition, the Stefan condition is applied at the phase change interfaces

$$
\mathrm{k}_{\mathrm{s}}^{*}\left(\mathbf{n} \cdot \nabla \mathrm{T}_{\mathrm{s}}\right)-(\mathbf{n} \cdot \nabla \mathrm{T})+\operatorname{PeSteV}_{\mathrm{p}}\left(\mathbf{n} \cdot \mathbf{e}_{\mathbf{z}}\right)=0
$$

where $T_{0}^{*}=\left(T_{0} / \Delta T\right), k_{s}^{*}=\left(k_{s} / k\right), C_{p s}^{*}=\left(C_{p s} / C_{p}\right)$.

The local magnetic fiel $d$ is simulated by the combination of the magnetic field produced by each electrical coil. The coil located at adjusted positions near the free surface circles the computation domain. The diameter of the coil is small and assumed to be zero for simpli- 
fication. The calculation of the magnetic field produced by the electrical coil is based on Biot-Savart's law. ${ }^{10}$

\section{Numerical Method}

The computation domain in the cylindrical coordinate system $(r, z)$ is transformed into the boundary-fitted curvilinear coordinate system ${ }^{11}(\eta, \zeta)$. The boundaryfitted curvilinear coordinate system is beneficial to track the phase change interface and the application of the boundary conditions. The transformed form of eq 2.3 is

$$
\begin{aligned}
\frac{\partial}{\partial \eta}\left(a_{i} \phi_{i} \frac{\partial \psi}{\partial \zeta}\right)- & \frac{\partial}{\partial \zeta}\left(a_{i} \phi_{i} \frac{\partial \psi}{\partial \eta}\right)+\frac{\partial}{\partial \eta}\left[\frac{b_{i}}{j}\left(g_{22} \frac{\partial c_{i} \phi_{i}}{\partial \eta}-g_{12} \frac{\partial c_{i} \phi_{i}}{\partial \zeta}\right)\right]+ \\
& \frac{\partial}{\partial \xi}\left[\frac{b_{i}}{J}\left(g_{11} \frac{\partial c_{i} \phi_{i}}{\partial \zeta}-g_{12} \frac{\partial c_{i} \phi_{i}}{\partial \eta}\right)\right]+d_{i j} J=0 \text { (3.1) }
\end{aligned}
$$

where

$$
\begin{aligned}
& r_{\zeta}=0, g_{11}=\left(\frac{\partial r}{\partial \eta}\right)^{2}+\left(\frac{\partial z}{\partial \eta}\right)^{2}, g_{22}=\left(\frac{\partial r}{\partial \xi}\right)^{2}+\left(\frac{\partial z}{\partial \xi}\right)^{2}, \\
& g_{12}=\left(\frac{\partial r}{\partial \eta}\right)\left(\frac{\partial r}{\partial \xi}\right)+\left(\frac{\partial z}{\partial \eta}\right)\left(\frac{\partial z}{\partial \xi}\right), J=\left(\frac{\partial r}{\partial \eta}\right)\left(\frac{\partial z}{\partial \xi}\right)-\left(\frac{\partial z}{\partial \eta}\right)\left(\frac{\partial r}{\partial \xi}\right)
\end{aligned}
$$

The according transformed boundary conditions in the melt zone are as follows.

(i) Along the growth axis:

$$
\psi=0, \omega=0, g_{22} \top_{\eta}-g_{12} \top_{\zeta}=0, g_{22} C_{\eta}-g_{12} C_{\zeta}=0
$$

(ii) Al ong the free surface:

$$
\begin{array}{r}
\left.\psi=0.5 \mathrm{~V}_{\mathrm{p}}, \omega=-\left(\frac{\mathrm{MaPr}}{\mathrm{Pe}} \frac{\partial \mathrm{T}}{\partial \zeta}\right)\right) \\
g_{22}^{1 / 2}, \mathrm{~g}_{22} \mathrm{C}_{\eta}-\mathrm{g}_{12} \mathrm{C}_{\zeta}=0-\frac{\mathrm{g}_{22} \mathrm{~T}_{\eta}-\mathrm{g}_{12} \mathrm{~T}_{\zeta}}{\mathrm{J} \mathrm{g}_{22}^{1 / 2}}= \\
\operatorname{Ra}\left[\left(\mathrm{T}+\mathrm{T}_{0}^{*}\right)^{4}-\left(\mathrm{T}_{\mathrm{a}}+\mathrm{T}_{0}^{*}\right)^{4}\right]
\end{array}
$$

(iii) Along the melting interface:

$$
\begin{aligned}
& \psi=0.5 \mathrm{~V}_{\mathrm{p}} \mathrm{r}^{2}=\omega=-\frac{\mathrm{g}_{11}}{\mathrm{r} \mathrm{J}^{2}} \frac{\partial^{2} \psi}{\partial \xi^{2}}, \mathrm{~T}=0, \frac{\mathrm{g}_{11} \mathrm{C}_{\zeta}-\mathrm{g}_{12} \mathrm{C}_{\eta}}{\mathrm{J} \mathrm{g}_{11}^{1 / 2}}= \\
& \mathrm{Pe}_{\mathrm{s}}(\mathrm{C}-1) \frac{\mathrm{r}_{\eta}}{\mathrm{g}_{11}^{1 / 2}}
\end{aligned}
$$

(iv) Along the growth interface:

$$
\begin{aligned}
\psi=0.5 \mathrm{~V}_{\mathrm{p}} \mathrm{r}^{2}, \omega=-\frac{\mathrm{g}_{11}}{\mathrm{r} \mathrm{J}^{2}} \frac{\partial^{2} \psi}{\partial \xi^{2}}, \mathrm{~T}=0, \\
\frac{\mathrm{g}_{11} \mathrm{C}_{\xi}-\mathrm{g}_{12} \mathrm{C}_{\eta}}{\mathrm{J} \mathrm{g}_{11}^{1 / 2}}=\mathrm{Pe}_{\mathrm{s}} \mathrm{V}_{\mathrm{p}}\left(1-\mathrm{k}_{0}\right) \mathrm{C} \frac{\mathrm{r}_{\eta}}{\mathrm{g}_{11}^{1 / 2}}
\end{aligned}
$$

The thermal boundary conditions for the solid phase can be transformed in the same way.

The transformed governing equations are solved on the uniform meshes in the transformed computation domain by the finite control-volume difference method. ${ }^{12}$ The "pseudo-time-dependent" method 13 is used to track the phase change interface. Tests were conducted to ensure that the numerical results were mesh-independent to within a tolerance of less than $1 \%$ (relative error)
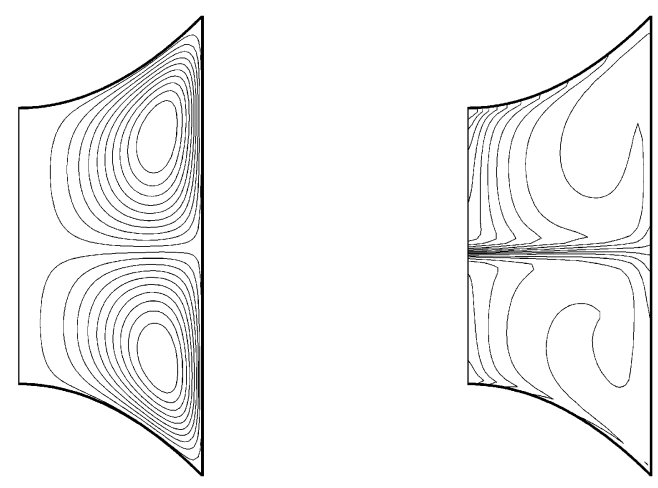

Figure 2. (a) Streamfunction and (b) impurity concentration distributions in the melt zone for the case without the applied magnetic field.

between two consecutively refined (node points doubled) meshes. The grid meshes of $41 \times 81,41 \times 61$, and $41 \times$ 81 were used in feed rod, melt zone, and crystal rod, respectively. Both the field variables and the interface position have converged within a preset relative tolerance of $1 \times 10^{-4}$. Numerical simulations were carried out for a wide range of conditions, and a selection of the computed results is presented bel ow. The physical properties of $\mathrm{P}$-doped $\mathrm{Si}^{14}$ used in the numerical simulation are listed in the Appendix.

\section{Results and Discussion}

To serve the comparison, the numerical results of the streamfunction and impurity concentration distributions for the case without the applied magnetic field are shown in Figure 2. It can be seen that without the applied magnetic field, the thermocapillary convection induced by the free surface tension gradient is relatively strong. The flow circulation nearly occupies the whole melt zone. The large streamfunction gradient appears in the region near the free surface because the driven force originates at the free surface. The flow field pattern induces the significant impurity concentration gradient at the curved growth interface. It will result in the detrimental lateral macrosegregation in the grown crystal. On the other hand, the heat transfer in the melt zone is dominated by heat conduction because of the small Pr number of Si melt. Thus, the flow field pattern changes will not alter the temperature distribution significantly and the temperature distribution study is neglected here. Figure 3 illustrates the streamfunction and concentration distributions for the cases with the longitudinal magnetic fiel $\mathrm{d}^{5}$ (Figure $3 \mathrm{a}$ ) and the local magnetic fields produced by two electrical coils located at selected positions $z=16.5$ and $z=1.71$ (Figure $3 b$ ) and $z=18.4$ (Figure 3c), respectively, and the damped flow vel ocities at the free surface are shown in Figure 4. The configurations of the local magnetic fields are shown in the previous studies. ${ }^{6,7}$ The computed results show that although the mechanisms are different, both the longitudinal magnetic fiel ds and the local magnetic fields can suppress the flow vel ocities at the free surface. For the configuration with free surface considered, the longitudinal magnetic field can directly damp the lateral flow velocity and then confine the thermocapillary convection developing area. Thus, the 

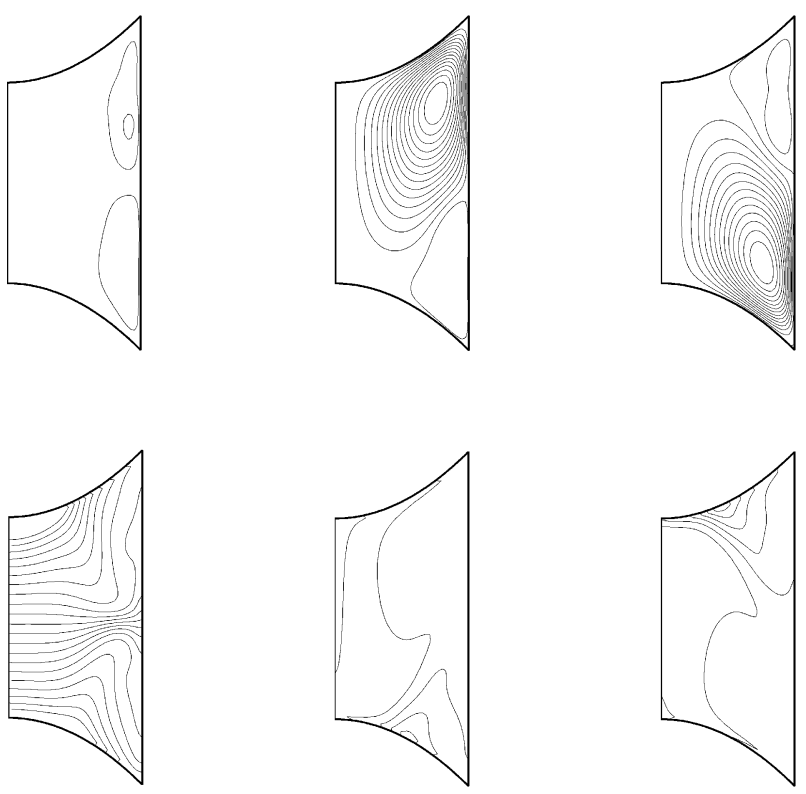

(a)

(b)

(c)

Figure 3. Streamfunction (upper) and impurity concentration (lower) distributions for the cases (a) with a longitudinal magnetic field and with the local magnetic fields produced by two el ectrical coils located at (b) $z=16.5, z=17.1$ and (c) $z=$ 18.0, $z=18.4$.

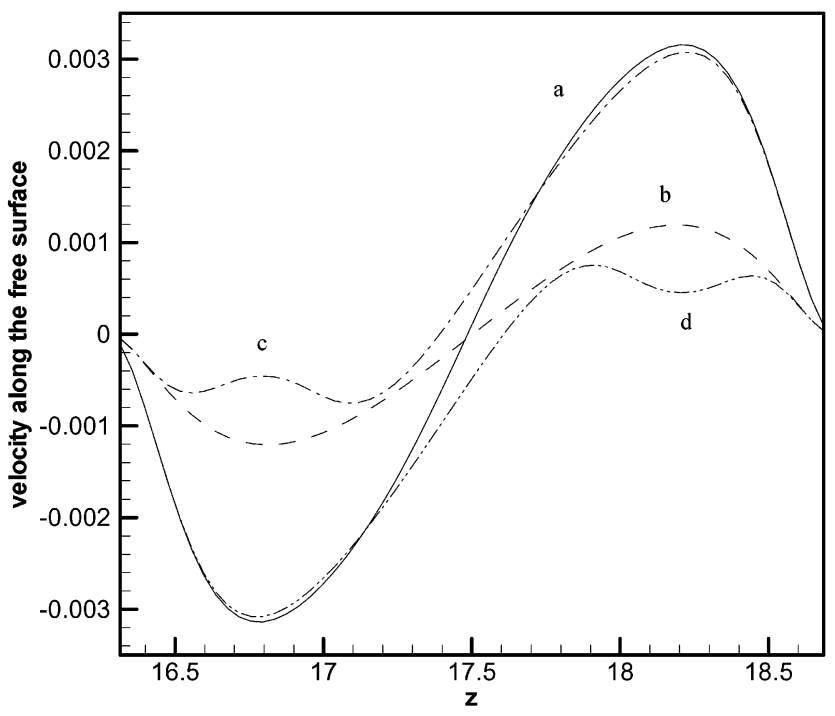

Figure 4. Flow velocity along the free surface in cases (a) without the magnetic field and (b) with the longitudinal magnetic field and with the local magnetic fields produced by two electrical coils located at (c) $z=16.5, z=17.1$ and (d) $z=$ $18.0, z=18.4$.

melt flow at the free surface is suppressed. However, the residual thermocapillary convection is still relatively strong in the region near the free surface as compared to the flow in the central region of the melt zone. Such a flow pattern results in the large impurity concentration nonuniformity at the growth interface (Figure 5). The local magnetic fields are designed to directly dampen the thermocapillary flow in the regions near the lower part and upper part of the free surface, respectively. By the effective reduction of the driving force origin, the thermocapillary convection in the whole melt zone is reduced as a result (F igure $3 b, c)$. Thus, the

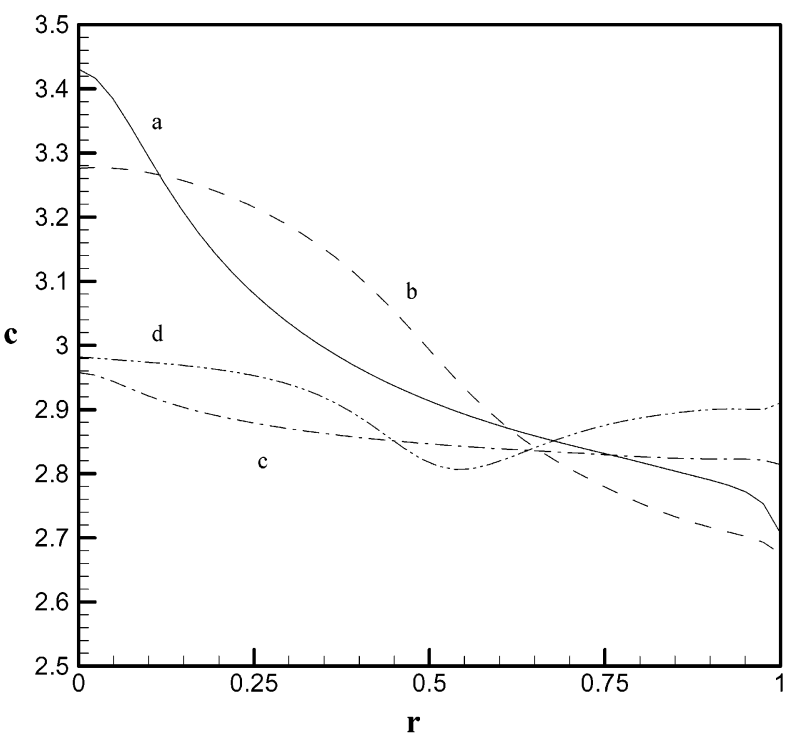

Figure 5. I mpurity concentration distributions at the growth interface in cases (a) without the magnetic field and (b) with the longitudinal magnetic field and with the local magnetic fields produced by two electrical coils located at (c) $z=16.5$, $z=17.1$ and $(d) z=18.0, z=18.4$. The $\xi$ values are, respectively, $24.5,19.8,7.0$, and $4.9 \%$.

residual flow field pattern is more uniform and beneficial to reduce the impurity concentration nonuniformity at the growth interface.

To quantitatively evaluate the influences of the magnetic field configurations on the impurity concentration distribution at the growth interface, the definition of the impurity concentration nonuniformity at the growth interface is introduced as follows

$$
\zeta=\frac{C_{\max }-C_{\min }}{C_{\text {avg }}}
$$

where subscripts max, min, and avg denote the maximum, minimum, and average values at the growth interface, respectively. The corresponding $\zeta$ values in Figure 5 are 24.5, 19.8, 7.0, and $4.9 \%$ for the cases without magnetic field, with the longitudinal magnetic field, and with the local magnetic fields, respectively. It can be seen that the flow field pattern of the case with the longitudinal magnetic field induces the large impurity concentration nonuniformity at the growth interface, and the local magnetic fields can reduce the impurity concentration nonuniformity at the growth interface significantly in comparison with the case of longitudinal magnetic field.

The influence of the local magnetic fields on the crystal growth in a floating zone with planar phase change interfaces has been discussed in the previous study. ${ }^{7}$ The impurity concentration distributions at the planar growth interface are shown in Figure 6 for cases without the applied magnetic field, with the longitudinal magnetic field, and with the local magnetic field produced by two electrical coils located at $z=1.4$ and 2.0. The related $\xi$ values are $8.3,6.7$, and $0.7 \%$, respectively. As compared with the computed results in Figure 5, it can be seen that the impurity concentration nonuniformity at the growth interface in the case with a curved 


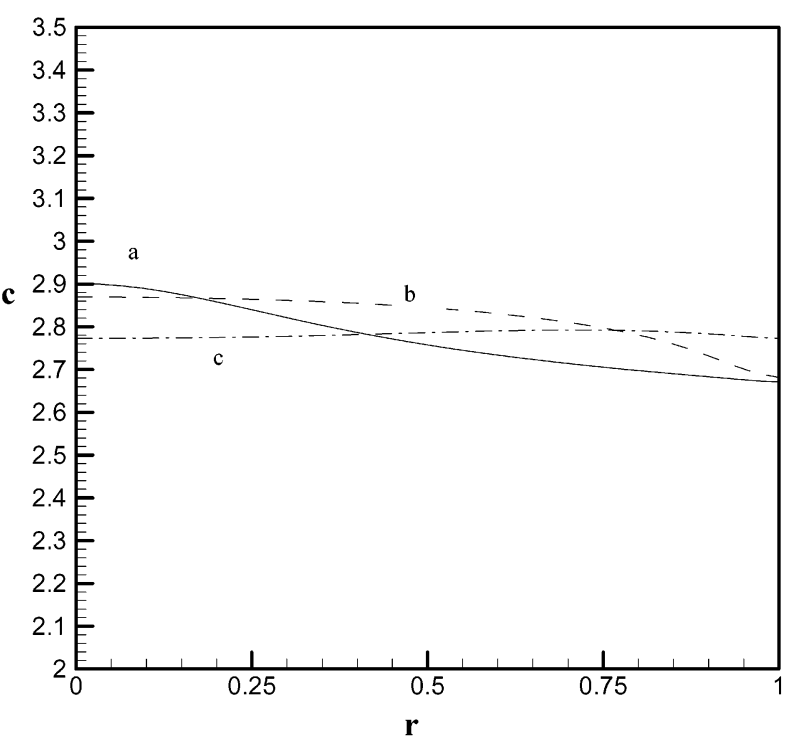

Figure 6. I mpurity concentration distribution at the planar growth interface in the cases (a) without the magnetic field, (b) with the longitudinal field, and (c) with the local magnetic field produced by two electrical coils located at $z=1.4, z=$ 2.0. The related $\zeta$ values are $8.3,6.7$, and $0.7 \%$, respectively.

phase change interface is much larger than the case with a planar phase change interface no matter with or without the presence of the applied magnetic fields. The comparison reveals that a relative planar interface is more beneficial to a uniform impurity concentration distribution at the growth interface, and the relative planar interface can be obtained by the proper heat control.

\section{Conclusion}

The time-dependent thermocapillary convection will induce the impurity concentration striations in the grown crystal. The applied magnetic fields can delay the onset of the oscillatory convection. However, the longitudinal magnetic field produces the large concentration nonuniformity at the growth interface. Applying the local magnetic field near the free surface can control the flow field and improve the impurity concentration distributions at the solidification interface. ${ }^{6,7}$ The influence of the local magnetic fields on the floating zone melting crystal growth is studied in the present paper. The computed results show that applying the local magnetic fields can significantly reduce the impurity concentration nonuniformity at the growth interface in comparison with the case of the longitudinal field. Moreover, the impurity concentration nonuniformity at the curved solidification interface is more serious than the case with the planar interface when the magnetic fields are applied. It illustrates that a planar growth interface is beneficial to a relatively uniform impurity concentration distribution at the growth interface in the crystal growth. It is known that the heating process is important in determining the solidification interface shape, so except for the typical ambient temperature distribution (2.1) for the modeling study of the floating zone process, other ambient temperature distributions are necessary to be further studied for searching a relative planar solidification interface.
Acknowledgment. The research is supported by the Project 95-yu-34 of the Ministry of Science and Technology of China and Grant 19789201 of the National Natural Science Foundation of China.

\section{Nomenclature}

$(r, z)$ : cylindrical coordinate system

$\left(B_{r}, B_{z}\right)$ : magnetic field components

$L$ : computation domain length

$\mathrm{R}_{0}$ : computation domain radius

$\mathrm{u}$ : $r$ direction velocity component

v: z direction velocity component

$\mathrm{T}$ : melt temperature

$\mathrm{T}_{\mathrm{s}}$ : solid temperature

$\mathrm{T}_{\mathrm{p}}$ : ambient temperature maximum

$\mathrm{T}_{\infty}$ : far end temperature

$\mathrm{T}_{0}$ : melting temperature of $\mathrm{Si}$

$\mathrm{T}_{\mathrm{a}}(\mathrm{z})$ : ambient temperature

a: ambient temperature distribution width

$\mathrm{V}_{\mathrm{p}}$ : crystal pulling speed

$v$ : kinematic viscosity

$\mathrm{k}$ : thermal conductivity

$\kappa$ : thermal diffusivity

$\sigma_{\mathrm{e}}$ : electrical conductivity

$\epsilon$ : heat emissivity

$\sigma$ : Stefan-Boltzmann constant

$\mathrm{C}_{0}$ : feed rod impurity concentration

C: melt impurity concentration

$\epsilon_{\mathrm{s}}$ : heat emissivity of solid phase

$\mathrm{k}$ : thermal conductivity of melt

$\mathrm{k}_{\mathrm{s}}$ : thermal conductivity of solid

$\mathrm{C}_{\mathrm{p}}$ : heat capacity of melt

$\mathrm{D}$ : impurity diffusivity

$\Delta \mathrm{H}$ : latent heat of $\mathrm{Si}$

$\sigma_{T}$ : surface tension gradient

$\mu_{\mathrm{r}}$ : relative magnetic permeability

$\mu_{0}$ : magnetic permeability of vacuum

$I_{0}$ : el ectric current intensity in coil

$\rho_{0}$ : density of pure $\mathrm{Si}$

$\mathrm{k}_{0}$ : segregation coefficient

$B_{0}$ : magnetic field magnitude scale

$\mathrm{U}_{0}$ : velocity scale

$\omega_{0}$ : vorticity scale

$\psi_{0}$ : steamfunction scale

$\Delta T$ : temperature scale

Pr: Prandtl number

Ste: Stefan number of melt

Ha: Hartmann number

Ra: radiation number of melt

$\mathrm{Ra}_{\mathrm{s}}$ : radiation number of solid

Pe: Peclet number

$\mathrm{Pe}_{\mathrm{s}}$ : impurity Peclet number

Ma: Marangoni number

$\mathbf{e}_{z}$ : unit vector $z$ direction

n: unit normal vector

\section{Appendix}

Physical properties of P-doped Si:

$\mathrm{T}_{0}=1688 \mathrm{~K}$

$\mathrm{T}_{\infty}=1643 \mathrm{~K}$

$\Delta \mathrm{T}=10 \mathrm{~K}$

$\Delta \mathrm{H}=1.803 \times 10^{6} \mathrm{~J} / \mathrm{kg}$

$\mathrm{R}_{0}=5.0 \times 10^{3} \mathrm{~m}$

$\mathrm{L}=1.75 \times 10^{2} \mathrm{~m}$

$\mathrm{a}=0.01 \mathrm{~m}$

$\rho_{0}=2.55 \mathrm{~g} / \mathrm{cm}^{3}$

$v=0.0035 \mathrm{~cm}^{2} / \mathrm{s}$

$\kappa=0.255 \mathrm{~cm}^{2} / \mathrm{s}$

$\mathrm{k}=0.64 \mathrm{~W} / \mathrm{cm} \mathrm{K}$

$\mathrm{k}_{\mathrm{s}}=0.22 \mathrm{~W} / \mathrm{cm} \mathrm{K}$

$\mathrm{C}_{\mathrm{p}}=1.038 \times 10^{3} \mathrm{~J} / \mathrm{kg} \mathrm{K}$

$\mathrm{C}_{\mathrm{ps}}=1.059 \times 10^{3} \mathrm{~J} / \mathrm{kg} \mathrm{K}$ 
$\sigma_{\top}=-1.3 \times 10^{-4} \mathrm{~N} / \mathrm{mK}$

$\mathrm{D}=2.7 \times 10^{-8} \mathrm{~m} 2 / \mathrm{s}$

$\mathrm{k}_{0}=0.35$

$\epsilon=0.3$

$\mu_{0}=4 \pi \times 10^{-7} \mathrm{H} / \mathrm{m}$

$\epsilon_{\mathrm{s}}=0.7$

$\sigma_{\mathrm{e}}=1.23 \times 10^{6} \Omega^{-1} \mathrm{~m}^{-1}$

$\mathrm{V}_{\mathrm{p}}=1.0 \times 10^{-5} \mathrm{~m} / \mathrm{s}$

\section{References}

(1) De Leon, N.; Guldberg, J .; Salling, J . J . Cryst. Growth 1981, $55,406-408$.

(2) Robertson, D. G., J r.; O'Connor, D. J . J . Cryst. Growth 1986, 76, 111-122.

(3) Series, R. W.; Hurle, D. T. J . J . Cryst. Growth 1991, 113, 305-328.

(4) Croll, A.; Dold, P.; Benz, K. W. J . Cryst. Growth 1994, 137, 95-101.
(5) Lan, C. W. J . Cryst. Growth 1996, 169, 269-278.

(6) Li, K.; Hu, W. R. J . Cryst. Growth 2001, 222, 677-684.

(7) Li, K.; Hu, W. R. J . Cryst. Growth 2001, 230, 125-134.

(8) Kim, D. H.; Adornato, P. M.; Brown, R. J . Cryst. Growth 1988, 89, 339-356.

(9) Lan, C. W. Int. J . Numer. Methods Fluids 1994, 19, 41-65.

(10) Smythe, W. R. In Static and Dynamic Electricity: Third Edition, Revised Printing; Richtmyer, F. K., DuBridge L. A, Eds.; Hemisphere Publishing Corporation: New York, 1989; pp 288-292.

(11) Thompson, J . F .; Thames, F. C.; Mastin, C. W. NASA Report, NASA-CR-2729, J uly 1977.

(12) Spalding, D. B. Int. J . Numer. Methods Eng. 1972, 4, 551562

(13) Xiong, B.; Hu, W. R. J . Cryst. Growth 1993, 133, 155-167.

(14) Lan, C. W.; Kou, S. J . Cryst. Growth 1991, 108, 351-366.

CG020029+ 